\title{
Prolonged acidosis is a feature of SGLT2i-induced euglycaemic diabetic ketoacidosis
}

\author{
Mohammed Faraz Rafey ${ }^{1,2}$, Arslan Butt ${ }^{1}$, Barry Coffey ${ }^{1}$, Lisa Reddington ${ }^{1}$, Aiden Devitt ${ }^{1}$, David Lappin ${ }^{1}$ and \\ Francis M Finucane ${ }^{1,2}$ \\ ${ }^{1}$ Galway University Hospitals, Galway, Ireland and 2HRB Clinical Research Facility, National University of Ireland \\ Galway, Galway, Ireland \\ Correspondence \\ should be addressed \\ to F M Finucane \\ Email \\ francis.finucane@hse.ie
}

\section{Summary}

We describe two cases of SGLT2i-induced euglycaemic diabetic ketoacidosis, which took longer than we anticipated to treat despite initiation of our DKA protocol. Both patients had an unequivocal diagnosis of type 2 diabetes, had poor glycaemic control with a history of metformin intolerance and presented with relatively vague symptoms post-operatively. Neither patient had stopped their SGLT2i pre-operatively, but ought to have by current treatment guidelines.

\section{Learning points:}

- SGLT2i-induced EDKA is a more protracted and prolonged metabolic derangement and takes approximately twice as long to treat as hyperglycaemic ketoacidosis.

- Surgical patients ought to stop SGLT2i medications routinely pre-operatively and only resume them after they have made a full recovery from the operation.

- While the mechanistic basis for EDKA remains unclear, our observation of marked ketonuria in both patients suggests that impaired ketone excretion may not be the predominant metabolic lesion in every case.

- Measurement of insulin, C-Peptide, blood and urine ketones as well as glucagon and renal function at the time of initial presentation with EDKA may help to establish why this problem occurs in specific patients.

\section{Background}

Sodium glucose-like transporter-2 inhibitor (SGLT2i) drugs have become widely prescribed for patients with type 2 diabetes (T2DM) because of their efficacy in improving glycaemic control (1), reducing cardiovascular and all-cause mortality (1) as well as improving renal (2) and cardiac (3) function in patients with chronic kidney disease and heart failure, respectively. Moreover, SGLT2is do not cause hypoglycaemia or weight gain, unlike some other diabetes medications. They inhibit the reabsorption of glucose from the renal filtrate, thus inducing glycosuria (4), which can commonly lead to genitourinary infections. In 2015, the first description of a rare and unanticipated complication of SGLT2i use, euglycaemic diabetic ketoacidosis (EDKA) was described, whereby patients with an unequivocal diagnosis of type 2 diabetes developed metabolic acidosis with very high levels of ketones consistent with DKA, but with normal glucose levels. While EDKA has been well described, with an incidence of approximately $0.1 \%$ in the EMPAREG (1) and CANVAS (5) randomised controlled trials $(1,5)$, its mechanistic basis is still not well established. It has been observed predominantly in hospitalised patients. Whether the duration of the metabolic acidosis is different from hyperglycaemic ketoacidosis has not been well described 
previously. We sought to describe our experience of the management of two patients with EDKA in whom the metabolic derangement took longer than expected to reverse.

\section{Case presentation}

Case 1: A 44-year-old white Irish male was found to have generalised weakness, lethargy, nausea and anorexia, 6 days post C5-C7 cervical decompression. He had a 5 -year history of type 2 diabetes, which was poorly controlled on dulaglutide $1.5 \mathrm{mg}$ subcutaneously weekly and canagliflozin $300 \mathrm{mg}$ once daily, with a HbA1c of $67 \mathrm{mmol} / \mathrm{mol}$. He also had dyslipidaemia, treated with atorvastatin $10 \mathrm{mg}$ nocte and ezetimibe $10 \mathrm{mg}$ nocte. He had a history of metformin intolerance. He had no other medical history of note. He drank less than 10 units of alcohol per week. He had an insulin-resistant phenotype, with acanthosis nigricans, axillary and cervical skin tags. His BMI was $38.8 \mathrm{~kg} / \mathrm{m}^{2}$. Five days earlier, on his first post-operative day, he had been noted by the anaesthetic team to have ketoacidosis in the intensive care unit with a pH of 7.27, ' $2+$ ' ketonuria, ' $2+$ ' glycosuria and a serum bicarbonate of $18.2 \mathrm{mmol} / \mathrm{L}$, but as his blood glucose was just $9.4 \mathrm{mmol} / \mathrm{L}$, this was attributed to starvation ketosis and was treated with intravenous dextrose and oral fluids. His diabetes medications had not been changed prior to his discectomy, which was uneventful.

On post-operative day 6 , he complained of ongoing generalised fatigue, lethargy and anorexia. He was apyrexial but tachypnoeic (24 breaths per minute) and tachycardic (110 beats per minute) and clinically dehydrated with dry mucous membranes. Neurological, respiratory, cardiac and abdominal examination were otherwise normal. Urine analysis showed 4+ ketones and $2+$ glycosuria. Point of care capillary blood ketones were elevated at $4.3 \mathrm{mmol} / \mathrm{L}$ (normal $<0.6$ ). His renal function was normal. His $\mathrm{pH}$ was 7.1 (7.35-7.45), $\mathrm{HCO}^{-}$was $4.8 \mathrm{mmol} / \mathrm{L}$ (22 to 28 ), glucose $9.4 \mathrm{mmol} / \mathrm{L}, \mathrm{pCO}_{2}$ was $2.0 \mathrm{KPa}(4.6-6)$ and lactate was $0.8 \mathrm{mmol} / \mathrm{L}(<0.8)$ with a high anion gap of $33.8 \mathrm{mEq} / \mathrm{L}$ (8-16), consistent with a metabolic acidosis with partial respiratory compensation. A diagnosis of EDKA induced by the SGLT2i canagliflozin was made.

Case 2: A 59-year-old white Irish female had generalised weakness, dyspnoea and pre-syncope 3 days after elective laparoscopic right partial nephrectomy for removal of a renal oncocytoma. She had a history of type 2 diabetes for 17 years which was poorly controlled with a HbA1c of $79 \mathrm{mmol} / \mathrm{mol}$, with established diabetic retinopathy, neuropathy and trans-metatarsal amputation of the left fourth and fifth toes, hypertension, obesity with a BMI of $39 \mathrm{~kg} / \mathrm{m}^{2}$, polycystic ovarian syndrome, fibromyalgia and depression. She had a history of intolerance to metformin and was a non-responder to GLP1 therapy. Her regular medication included insulin glargine 32 iu daily, insulin aspart 12, 11 and 9 units with meals, duloxetine $120 \mathrm{mg}$ once daily, lercanidipine $10 \mathrm{mg}$ once daily, doxazosin $4 \mathrm{mg}$ once daily, ramipril $10 \mathrm{mg}$ once daily, pregabalin $350 \mathrm{mg}$ once daily, and 5 months prior to this episode, she was started on empagliflozin $25 \mathrm{mg}$ once daily. She drank less than 10 units of alcohol per month. On examination she was apyrexial, tachypnoeic ( 24 breaths per minute) and tachycardic (108 beats per minute) with a blood pressure of $105 / 55 \mathrm{mmHg}$ and was clinically dehydrated with dry mucous membranes. Neurological, respiratory, cardiac and abdominal examination were otherwise normal. Urine analysis showed ' $3+$ ' ketonuria and ' $2+$ ' glycosuria. Her renal function had been normal pre-operatively but her creatinine rose from 52 to $94 \mu \mathrm{mol} / \mathrm{L}$ (estimated glomerular filtration rate $58 \mathrm{mmol} /$ $\min 1.73 / \mathrm{m}^{2}$ ) on the first post-operative day, with mild, transient pre-renal acute kidney injury. Blood glucose was $12.3 \mathrm{mmol} / \mathrm{L}$. Point of care capillary blood ketones were elevated at $4.8 \mathrm{mmol} / \mathrm{L}$. Lactate was $0.5 \mathrm{mmol} / \mathrm{L}$, pH 7.23 and serum bicarbonate $9.3 \mathrm{mmol} / \mathrm{L}$, with a high anion gap of $32.6 \mathrm{mEq} / \mathrm{L}$. A diagnosis of EDKA induced by the SGLT2i empagliflozin was made.

\section{Investigation}

The investigations undertaken are reported in the 'Case presentation' section.

\section{Treatment}

Case 1: This patient was managed according to our DKA protocol, with aggressive intravenous fluid resuscitation, although with a much lower insulin dose than standard, starting at one unit of intravenous insulin per hour. His hydration status, fluid balance, electrolytes, glucose, capillary blood ketones, venous bicarbonate and $\mathrm{pH}$ were monitored closely. He remained acidotic at $24 \mathrm{~h}$ (Fig. 1), with a $\mathrm{pH}$ of 7.28 , bicarbonate of $15.4 \mathrm{mmol} / \mathrm{L}$ and blood ketones $2.1 \mathrm{mmol} / \mathrm{L}$. He was transferred to the ICU. At $40 \mathrm{~h}$, despite ongoing intravenous fluids, intravenous insulin and introduction of basal insulin, he remained acidotic with a $\mathrm{pH}$ of 7.29 , bicarbonate of $19.3 \mathrm{mmol} / \mathrm{L}$ and ketones of $0.8 \mathrm{mmol} / \mathrm{L}$. It was not until $92 \mathrm{~h}$ after initiation of therapy that the metabolic derangement 


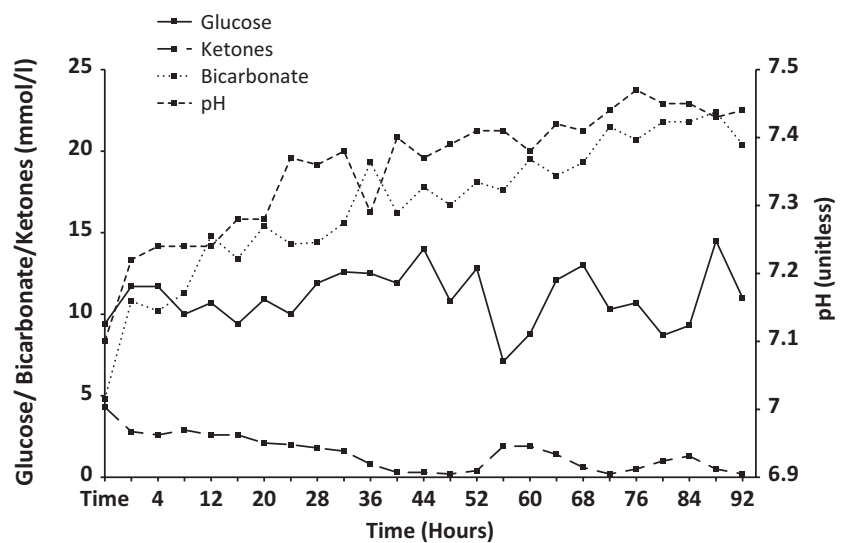

Figure 1

Changes in glucose, ketones, $\mathrm{pH}$ and bicarbonate in patient \#1.

normalised, with blood ketones dropping to $<0.6 \mathrm{mmol} / \mathrm{L}$ at that time. He was discharged home well the following day on insulin glargine 6 units once daily and once weekly dulaglutide.

Case 2: In a similar way, this patient was managed according to our DKA protocol, with aggressive intravenous fluid resuscitation and monitoring of her hydration status, fluid balance, electrolytes, glucose, capillary blood ketones, venous bicarbonate and $\mathrm{pH}$, with low-dose intravenous insulin (Fig. 2). Her renal function normalised quickly. At $28 \mathrm{~h}$ post initiation of the DKA protocol, her blood ketones were still elevated at $0.9 \mathrm{mmol} / \mathrm{L}$, with persisting low bicarbonate of $18.8 \mathrm{mmol} / \mathrm{L}$, but the DKA protocol was stopped as her $\mathrm{pH}$ and glucose levels had normalised. However, within $16 \mathrm{~h}$ her blood ketones increased again to $1.2 \mathrm{mmol} / \mathrm{L}$ and her $\mathrm{HCO}_{3}{ }^{-}$decreased to $17.4 \mathrm{mmol} / \mathrm{L}$. The DKA protocol was restarted immediately with a view to maintaining the intravenous fluids for a further $72 \mathrm{~h}$. Again, it was only after $92 \mathrm{~h}$ on the DKA protocol that the ketoacidosis had

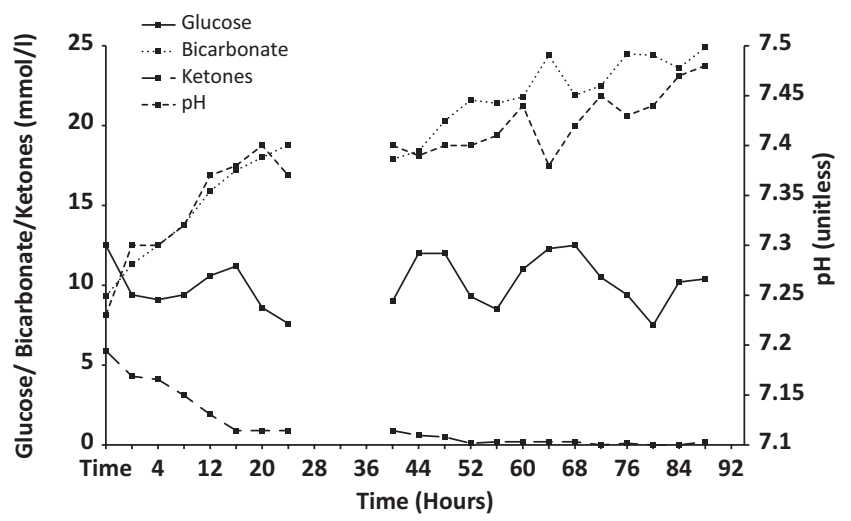

Figure 2

Changes in glucose, ketones, $\mathrm{pH}$ and bicarbonate in patient \#2. fully resolved, with a $\mathrm{pH}$ of 7.48, a normal anion gap, blood ketones of $0.2 \mathrm{mmol} / \mathrm{L}$ and serum bicarbonate of $24.9 \mathrm{mmol} / \mathrm{L}$. Then, mealtime subcutaneous insulin was initiated along with intermediate acting insulin glargine. The patient had further post-operative complications including an episode of bowel obstruction and had a protracted in-hospital stay. She was eventually discharged home on mealtime insulin aspart 9 units tds and insulin glargine 22 units daily.

\section{Outcome and follow-up}

Case 1: Four weeks after discharge on insulin glargine and dulaglutide, the patient was noted to have recurrent hypoglycaemia. His insulin was stopped and gliclazide MR $30 \mathrm{mg}$ was started, with subsequent excellent glycaemic control. However, over the following 18 months he required an increased dose of gliclazide MR to $120 \mathrm{mg}$ daily and the introduction of sitagliptin $100 \mathrm{mg}$ od in order to maintain glycaemic control.

Case 2: After discontinuation of empagliflozin and the introduction of the basal bolus insulin regime, the patient was followed after discharge for dose titration and achieved good glycaemic control on insulin glargine 24 units daily and insulin aspart 10 units with meals, with a HbA1c of $54 \mathrm{mmol} / \mathrm{mol}, 6$ months after discharge.

\section{Discussion}

While both patients ultimately responded well to intravenous fluids and insulin as expected, we were struck by the protracted and prolonged nature of their acidosis, which took $92 \mathrm{~h}$ to resolve in both cases. In a recent audit of our experience of treating 74 consecutive cases of DKA, the mean time to resolution of acidosis was $35 \mathrm{~h}$, consistent with findings in other centres $(6,7)$. The longer duration of acidosis in EDKA is not emphasised in current management guidelines and has not been widely described in the literature $(6,7)$. A recent cohort study in Korea noted that the acidosis took twice as long to resolve in EDKA as it did for DKA (4 vs 2 days) (8). We think that this critically important observation warrants reporting in other populations as clinicians need to be aware of the protracted nature of EDKA. Both of our patients were of white Irish ancestry. Our patients had a number of other features in common. Both had body mass indices in the range of severe obesity, both had an unequivocal diagnosis of type 2 diabetes which in both cases was poorly controlled on a background of intolerance to metformin. Both patients were diagnosed with EDKA post-operatively 
and presented with relatively vague symptoms. It is not clear how these observations might shed light on the aetiology of EDKA. Several theories exist in this regard.

Some suggest that SGLT2i drugs may impair the excretion of urinary ketones, but our (and others') observation of marked ketonuria would make it unlikely that this is the dominant metabolic lesion. We previously speculated that SGLT2i drugs may cause severe, abrupt but transient impairment in beta cell glucose sensing, leading to temporary but absolute beta cell failure and insulin deficiency, as is seen with glucotoxicity in ketosis prone ('Flatbush') diabetes (9). However, our second patient was on a basal bolus insulin regime at the time that her EDKA occurred, suggesting this mechanism is unlikely to apply to all patients, if any.

SGLT2i are known to stimulate alpha cell glucagon secretion which can diminish beta cell insulin production (10). This may have contributed to EDKA in our cases, but as we did not measure insulin or glucagon, we can only speculate. Regardless of the mechanism, treatment of EDKA requires the same approach as hyperglycaemic DKA, albeit with less insulin. An important distinction, though, is the use of dextrose rather than saline, given that these patients rarely have blood glucose levels great than $11 \mathrm{mmol} / \mathrm{L}$ and this offers an important therapeutic opportunity to administer a larger dose of intravenous insulin, in order to suppress ketosis. With the benefit of hindsight and in line with recent guidelines (7), our patients would ideally have stopped these drugs in the days preceding surgery. After these cases, our institution has introduced a policy of advising surgical patients to stop SGLT2i therapy $48 \mathrm{~h}$ before their procedure and re-introduce them a minimum of $48 \mathrm{~h}$ afterwards.

Future studies on the mechanistic basis for and predictors of EDKA ought to consider measurement of blood and urine ketones, as well as insulin, C-Peptide and glucagon levels at the time of presentation and again once the drug has stopped and metabolic recovery has occurred, in order to determine which metabolic lesions are associated with this uncommon but potentially serious iatrogenic complication of SGLT2i use.

\section{Declaration of interest}

F M F has received honoraria, travel grants, unrestricted educational grants and served on advisory boards for Novo Nordisk, Eli Lilly, Pfizer Inc., Sanofi-Aventis, Astra Zeneca, Merck Sharp and Dohme, Boehringer Ingelheim, Janssen and Novartis. David Lappin has received speakers' fees from Astra Zeneca, Boehringer Ingelheim and Lilly Pharmaceuticals and has received educational support from Mundi Pharma. Mohammed Faraz Rafey, Arslan Butt, Barry Coffey, Aiden Devitt and Lisa Reddington have no conflicts of interest to declare.

\section{Funding}

F M F is funded in part by a Clinical Research Career Development Fellowship from the Saolta Healthcare Group within the Health Service Executive, Ireland. Otherwise, this research did not receive specific grant funding from any agency in the public, commercial or not-for-profit sector.

\section{Patient consent}

Written informed consent for publication of their anonymised clinical details was obtained from both patients.

\section{Author contribution statement}

M F Rafey is an endocrinology registrar who was involved in the diagnosis and management of both cases and in writing the case reports. A Butt is a nephrology registrar who helped with the literature review and the writing of the case reports. $L$ Reddington is a diabetes nurse specialist who managed the patients and helped write the case reports. B Coffey was an orthopaedic intern and A Devitt the consultant orthopaedic surgeon who cared for the first patient and helped to write the manuscript. D Lappin is a consultant nephrologist who helped with drafting and reviewing the manuscript. F M Finucane is a consultant endocrinologist who supervised the endocrine care of these patients, as well as the collation of data and the drafting of the manuscript.

\section{References}

1 Zinman B, Wanner C, Lachin JM, Fitchett D, Bluhmki E, Hantel S, Mattheus M, Devins T, Johansen OE, Woerle HJ et al. Empagliflozin, cardiovascular outcomes, and mortality in Type 2 diabetes. New England Journal of Medicine 2015373 2117-2128. (https://doi. org/10.1056/NEJMoa1504720)

2 Perkovic V, de Zeeuw D, Mahaffey KW, Fulcher G, Erondu N, Shaw W, Barrett TD, Weidner-Wells M, Deng H, Matthews DR et al. Canagliflozin and renal outcomes in type 2 diabetes: results from the CANVAS Program randomised clinical trials. Lancet Diabetes and Endocrinology 20186 691-704. (https://doi.org/10.1016/S22138587(18)30141-4)

3 Wiviott SD, Raz I, Bonaca MP, Mosenzon O, Kato ET, Cahn A, Silverman MG, Zelniker TA, Kuder JF, Murphy SA et al. Dapagliflozin and cardiovascular outcomes in Type 2 diabetes. New England Journal of Medicine 2019380 347-357. (https://doi.org/10.1056/ NEJMoa1812389)

4 Burke KR, Schumacher CA \& Harpe SE. SGLT2 inhibitors: a systematic review of diabetic ketoacidosis and related risk factors in the primary literature. Pharmacotherapy 201737 187-194. (https:// doi.org/10.1002/phar.1881)

5 Neal B, Perkovic V, Mahaffey KW, de Zeeuw D, Fulcher G, Erondu N, Shaw W, Law G, Desai M, Matthews DR et al. Canagliflozin and cardiovascular and renal events in Type 2 diabetes. New England Journal of Medicine 2017377 644-657. (https://doi.org/10.1056/ NEJMoa1611925)

6 Danne T, Garg S, Peters AL, Buse JB, Mathieu C, Pettus JH, Alexander CM, Battelino T, Ampudia-Blasco FJ, Bode BW et al. International consensus on risk management of diabetic ketoacidosis in patients With Type 1 diabetes treated With sodium-glucose cotransporter (SGLT) inhibitors. Diabetes Care 201942 1147-1154 (https://doi.org/10.2337/dc18-2316)

7 Handelsman Y, Henry RR, Bloomgarden ZT, Dagogo-Jack S, DeFronzo RA, Einhorn D, Ferrannini E, Fonseca VA, Garber AJ, Grunberger $\mathrm{G}$ et al. American Association of Clinical 
Endocrinologists and American College of endocrinology position statement on the association of SGLT-2 inhibitors and diabetic ketoacidosis. Endocrine Practice 201622 753-762. (https://doi. org/10.4158/EP161292.PS)

8 Jeon JY, Kim SK, Kim KS, Song SO, Yun JS, Kim BY, Kim CH, Park SO Hong S, Seo DH et al. Clinical characteristics of diabetic ketoacidosis in users and non-users of SGLT2 inhibitors. Diabetes and Metabolism 2019. (https://doi.org/10.1016/j.diabet.2019.01.001)
9 Finucane FM. SGLT2 inhibitor-induced euglycaemic diabetic ketoacidosis may be due to abrupt, severe and transient impaired glucose sensing in susceptible individuals with a hitherto unrecognised beta cell SGLT variant. Medical Hypotheses 2018114 11-12. (https://doi.org/10.1016/j.mehy.2018.02.025)

10 Taylor SI, Blau JE \& Rother KI. SGLT2 inhibitors may predispose to ketoacidosis. Journal of Clinical Endocrinology and Metabolism 2015 100 2849-2852. (https://doi.org/10.1210/jc.2015-1884)

Received in final form 29 August 2019

Accepted 6 September 2019 